DESY M-89-22

October 1989

\title{
Impedance Calculation with URMEL-I Using Multigrid Methods
}

\author{
U. van Rienen \\ Deutsches Elektronen-Synchrotron DESY, Hamburg \\ T. Weiland \\ Technische Hochschule Darmstadt
}


DESY M-89-22

Oktober 1989

\title{
Impedance Calculation with URMEL-I \\ Using Multigrid Methods
}

\author{
Ursula van Rienen \\ Deutsches Elektronen-Synchrotron DESY, \\ Notkestr. 85, D-2000 Hamburg 52, Germany
}

Thomas Weiland

Technische Hochschule Darmstadt, Fachbereich 18,

Fachgebiet Theorie Elektromagnetische Felder,

Schloßgartenstr. 8, D-6100 Darmstadt, Germany

Talk presented at the COMPUMAG Conference on the Computation of Electromagnetic

Fields, Tokyo, Japan, Sept. 3-7, 1989 


\title{
Impedance Calculation with URMEL-I Using Multigrid Methods
}

\author{
Ursula van Ricnen \\ Deutsches Elektroncn-Synchrotron. DESY, Notkestr. 85, D-2000 Ham.burg 52, Germany \\ Thomas Weiland \\ Technische Hochschule Darmstadt, Fachbereich 18, Fachgebiet Theoric Elehtromagnetischer Felder, \\ Schloßgart.enst.r. 8, D-6100 Darmstadt, Gcrmany
}

\section{$A B S T R A C T$}

The computer code URMEL-I calculates the impedance of cylindrically symmetric obstacles of arbitrary shape with open side tubes. This code is specifically designed for the impedance calculation above cut-off where existing resonat or codes cannot be used. The numeric solution is based on the FIT-method, a finite difference method. It requires the solution of a large, inhomogeneous, complex matrix equation for a given frequency. A multigrid algorithm has been set up to solve the linear system, which is non-hermitian and indefinite. This specia] solver is described in this paper and results for different resonators are presented.

\section{INTRODUCTION}

A numerical method is presented for calculating the longitudinal impedance of a cylindrically symmetric structure, e.g. a cavity with beam ports in an accelcrator.

For a cavity with open side tubes made from perfectly conducting material the spectrum of the impedance differs qualitatively in the frequency range below and above the lowest cut-off frequency for travelling waves in the tube. The spectrum of the real part is discrete below cut-off and continuous above. Above the cut-off frequency no ideal resonance can exist. because energy is lost as fields travel out of the cavity. A peak in the spectrum above cut-off can be considered a resonance with finite quality factor.

There are a number of computer codes for evaluating the lowest resonant frequencies and quality factors for cavities which are needed for the impedance calculation below cut-off, e.g. [1], [2]. URMEL-I is based on URMEL [1] but calculates the impedance as a function of frequency (including the region above cut-off). For this calculation the cavity is excited by a current on the axis. An "open boundary condition" is implemeted in URMEL-I. A different approach for the treatment of the boundary conditions is used by Gluckstern and Neri, who adapted the resonator code SUPERFISH [3] for impedance calculations above cut-off [4].

\section{THE LONGITUDINAL IMPEDANCE}

The longitudinal impedance of a structure that has been excited by a current on the axis is given by:

$$
Z(\omega)=\frac{1}{q} \int_{-\infty}^{\infty} \dot{E}_{z}(r=0, \varphi=0, z, \omega) c^{i k z} d z,
$$

with the charge $g$, the wave number $k=\underline{\omega}$ and where $\tilde{E}_{z}$ is the Fourier transformation of the longitudinal electric fic]d $E_{z}$. ([5] gives more details.)

In $[6]$ it is shown that the integral can be taken at any radius $r$ for a cylindrically symmetric structure. Thus integrating at the tube radius gives the same result as the integral on the axis. The advantage of integrating at the tube radius is that the integrand vanishes in the tube region. Consequently one need only perform the integral over the gap of the cavity.

\section{NUMERICAL FORMULATION OF THE PROBLEM}

Maxwell's equations for the problem read as

$$
\oint_{\partial A} \vec{H}^{\prime} \cdot d \vec{s}=i \frac{\omega}{c} \cdot \int_{A} \epsilon_{r} \vec{E}^{\prime} \cdot d \vec{A}+I^{\prime}
$$

$$
\int_{i, A} \vec{F}^{\prime} \cdot d \vec{s}=-i \frac{u^{\prime}}{c} \cdot \int_{A} \mu, \vec{H}^{\prime} \cdot d \vec{A} .
$$

where $\partial A$ stands for the boundary of area $\mathrm{A}$ and $\vec{E}^{\prime}$ and $\vec{H}^{\prime}$ are normalized complex phasors (comparc [5]). At present only monopole fields are treated $(\partial / \partial \varphi=0)$.

They are discretized on a staggered rectangular grid by a special finite difference method, the FIT-method [7]. This implies many analogies with URMEL [1].

The magnetic field $H^{\prime}$ can be decomposed into an inhomogeneous part

$$
H_{\varphi}^{*}(\omega, r, z)=\frac{c g}{2 \pi r} c^{-i k z} .
$$

that is caused by the current, and a homogeneous part $H_{4}^{0}$

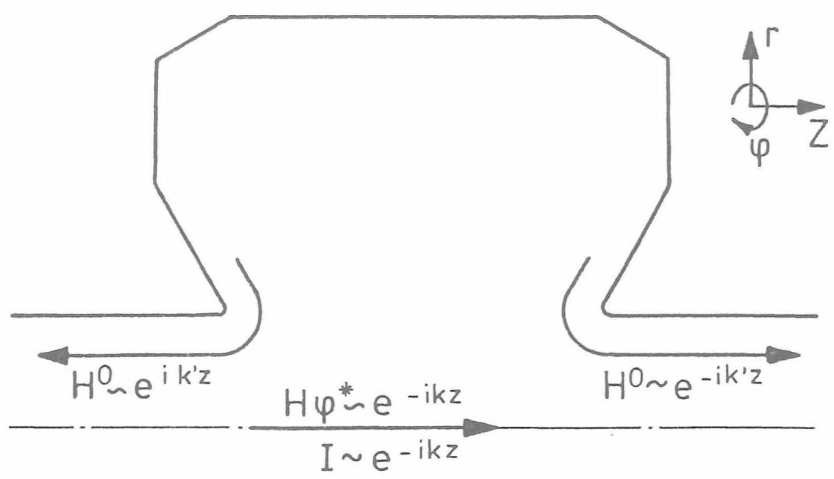

Figure 1: Longitudinal dependance of exciting current and magnetic field components.

For the numerical calculation the (infinitely long) tubes have to be cut at some convenient distance from the cavity. At these boundaries the reality of an infinitely long tube has to be simulated.

From ( 4 ) it follow's for $H_{\varphi}^{*}$

$$
H_{\psi}^{*}(\omega, r, z-\Delta)=H_{\varphi}^{*}(\omega, r, z) c^{i k \Delta} \doteq(1+i k \Delta) H_{\varphi}^{*}(\omega, r, z)
$$

For frequencies above cut-off the fields excited can propagate in the tube with propagation constant $k^{\prime}$. (compare figure 1 ). For the homogeneous azimuthal magnetic field the relation

$$
\begin{aligned}
& H_{\varphi}^{0}(\omega, r, z-\Delta)=H_{\varphi}^{0}(\omega, r, z) c^{i k^{\prime}(-\Delta)} \doteq\left(1-i k^{\prime} \Delta\right) H_{\varphi}^{0}(\omega, r, z) \\
& H_{\varphi}^{0}(\omega, r, z+\Delta)=H_{\varphi}^{0}(\omega, r, z) c^{-i k^{\prime} \Delta} \doteq\left(1-i k^{\prime} \Delta\right) H_{\varphi}^{0}(\omega, r, z)
\end{aligned}
$$

is valid in the left resp. right tube. $k^{\prime}$ is exactly evaluated in the frequency range just above cut-off where only one type of wave can propagate, for the higher frequencies the approximation $k^{\prime}=k$ is used. These first order equations are used to set up the difference equations for grid points at the right and left boundaries.

For the equations for $E_{r}$ at the left and right boundaries of the grid the open boundary simulation is used (compare (5), (6). ( 7)). The difference equations for $E z$ use (for $r>0)$ the rclation $r H^{*}(\omega, r, z)=$ $(r-\Delta) I_{\varphi}^{*}(\omega, r-\Delta, z)$ which holds because of $(4)$; on the axis $(r=0)$ the current dues not vanish but can be expressed in terms of $H_{c}^{*}$. In the difference equation for $H_{\varphi}^{0}$ the other components are each replaced by their difference equations. 
This yields a swetem of lmear equations with the homengeneruse azinuthal field components as unknowns and the inlomogeneous ones on the right hand sides.

The FIT-method uses the area of a grid cell and the lengths of the sides for the discretization. An extension of the FIT-method was adopted in connection with the solutjon of the resulting linear algebraic problem: When a cell is in any way partially filled with metal, only the area of the remaining vacuum and the lengths of the sides not bordering metal are used.

\section{SOLUTION OF THE NUMERICAL PROBLEM BY A MULTJGRID ALGORITHM}

The discretization of Maxwell's equations leads to a complex linear system of equations

$$
\mathrm{L} u=f
$$

with $\mathbf{L}=\mathbf{A}+i k^{\prime} \mathbf{D}-k^{2} \mathbf{I}, \mathbf{u}=\left(H_{\varphi 1}^{0}, \ldots, H_{\varphi N}^{0}\right)^{T}, \mathbf{f}=k^{2}\left(H_{\varphi 1}^{*}, \ldots, H_{\varphi N}^{*}\right)^{T}$.

$\mathrm{L}$ is a $(\mathrm{N} \times \mathrm{N})$-matrix and has a band structure with only four offdiagonals. $\mathrm{L}$ is non-hermitian and not even positive definite. Near (quasi-)resonances $\mathrm{L}$ even becomes nearly singular. At resonances below cut-off $\mathrm{L}$ is exactly singular. Because of round-off errors this is also true near these resonances.

The large, sparse linear system ( 8 ) has to be solved for each given frequency. As the usual iterative methods either do not work for indefinite, non-hermitian systems or have a poor performance, a multigrid algorithm has been developed to solve the linear system.

Multigrid methods [8] have been very successful in the fast solution of partial differential equations. The main idea of the multigrid method lies in the combination of an iteration method $I$, which smoothes the high frequency part.s of the error in a few steps, with another il.cration $I I$, that reduces the low frequency parts in the error. $I$ is just a classical iteration method like the Gauss-Seidel method. One iteration step of $I I$ consists of a correction evaluated on a coarser grid. Even though $I I$ is not convergent the combination of $I$ and $I I$ converges very fast. The iteration steps of $I I$ are relatively fast because the problems on the coarser grids have fewer unknowns.

In URMEL-I a so called Full-Multi-Grid-Method (FMG) with Vcycles is used. A V-cycle is a special kind of single iteration step of II. Figure 2 shows schematically on two grids how this works. In practice at least three grids are usually taken.

The multigrid algorithm in URMEL-I consists of the following components:

- The coarser grids are given by standard coarscning of the grid on the next higher level, i.e. $h_{\text {coarse }}=2 h_{\text {finc }}$ for regular grids.

- On each grid level the matrix $L_{i}$ has to be set. The compatible coarsening is chosen: i.e. $L_{2}$ is set up by the FIT-method like $\mathbf{L}$ on the finest grid. In this process $\mathrm{it}$ is very important to solve the same physical problem, i.c. to treat the same geometry. Therefore the extension of the usual discretization by the FIT. method on a rectangular grid, which is described above, was introduced.

Since discretization shifts the eigenvalues of the Laplace-operator the eigenvalues of the indefinite operator $\Delta-k^{2} l$ may change their sign on the coarser grids. An appropriate shift-factor for $k$ and $k^{\prime}$ was introduced t.o correct the discrete cigenvalues.

- As grid transfer the bilinear interpolation and the corsesponding restriction are chosen. Rather than a pure bilinear interpolation. an undcr-intcrpolation with the squared shift factor is used.

- As relaration method the Gauss-Seidel method is taken as long as a $\mathrm{V}$-cycle reduces the $\|\cdot\|_{2}$-Norm of the residuum well enough. From then on the Kaczmarz method [9] is used.

- The number of $\mathrm{V}$-cycles has not been fixed a priori but is set by a convergence criterium. Also the numbcr of relaxation succps depends on a convergence criterium.
- The solution on the coarsest grid is done by LUT-factorization with a LINPACK-routine [10].

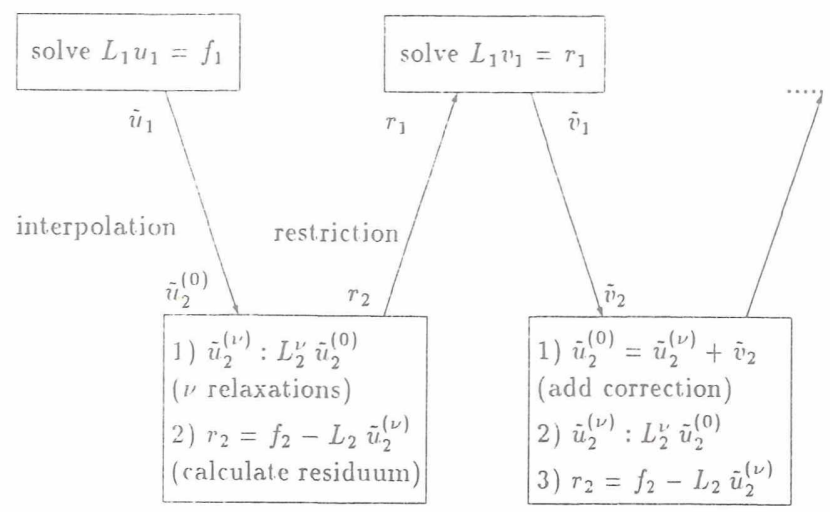

Figure 2: FMG-method on two grids.

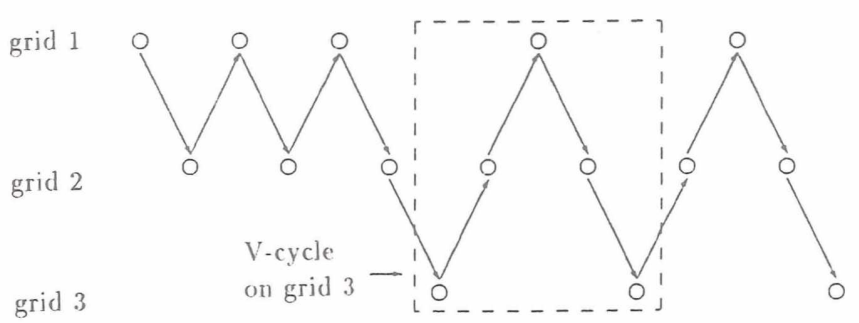

Figure 3: FMG-method with three grids and two V-cycles on each grid. Grid 1 is the coarsest grid.

Except in the vicinity of frequencies for which $\mathrm{L}$ becomes (nearly-) singular, the convergence of the multigrid algorithm in URMEL-I is good. Comparisons with a direct solution method were only possible for small problcms but there good agreement was obtained between results from the direct solution method and the multigrid solver. In conclusion it can be said that the results are satisfying and show a seasonable agreement with quasi analytical results and results obtained from wakepotential calculations with TBCI [6].

\section{EXAMPLES}

The longitudinal impedance and quality factors for several cavities are shown. The reader interested in studies of the convergence properties of the multigrid algorithm is referred to [11] where the whole subject. of URMEL-I and the multigrid algorithm is treated in detail.

As first example a pill-box with $65 \mathrm{~mm}$ gap, $100 \mathrm{~mm}$ radius and 50 mm tube radius is chosen.

For pill-boxcs with side tubes the impedance can be analytically calculated by Fourier series. H.Henke [12] used this to compute the impedance for pill-bores numerically. Figure 4 shows the impedance calculated with the LU-factorization as solver for $\Delta_{z}=4.06 \mathrm{~mm}, 8.12$ $\mathrm{mm}$ and $16.25 \mathrm{~mm}$ giving 640,160 and 40 vacuum cells (respectively) and the results by Honke's semi-analytical method. Good convergence is observable for $\Delta_{z} \rightarrow 0$. The use of the shift-factor prevents a compression of the impedance curve in the frequency direction which occurs without this eigenvalue correction (compare [11]).

In the following the effectiveness of the special measures undertaken to handle the eigenvalue shift is demonstrated using the pill-bor as an example. As mentioned, the eigenvalue shift due to discretization is estimated and a shift-factor is used so that the problem $L^{s} u=f^{s}$ with $\mathbf{L}^{s}=\mathbf{A}+i k^{\prime} \mathbf{D}-s \cdot k^{2} \mathbf{I}$ and $\mathbf{f}^{s}=s \cdot \mathfrak{f}$ is solved instead of $\mathrm{L} \mathbf{u}=\mathrm{f}$. 


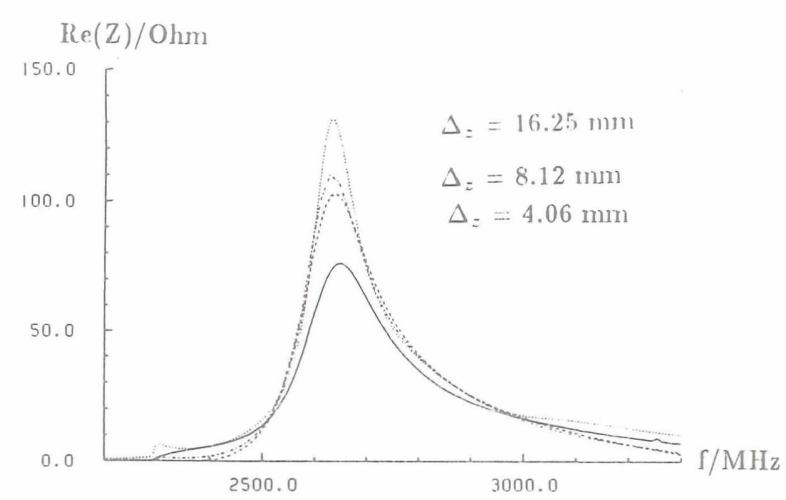

Figure 4: Real part of the impedance above cut-off $(=2295 \mathrm{MHz})$ calculated for the pill-box by URMEL-I with direct solution method and stepsizes $\Delta_{z}=4.06,8.12,16.25 \mathrm{~mm}$ and impedance by semi-analytical solution with Henke's code.

Figures 5 and 6 show the ten lowest eigenvalues of $\mathrm{L}$ resp. $\mathrm{L}^{\mathrm{s}}$ for $f=k \cdot \frac{c}{2 \pi}=3500 \mathrm{MHz}$ on the grids $G_{2}$ and $G_{1}$. The grid $G_{1}$ has the stepsizes $\Delta_{r}=25 \mathrm{~mm}, \Delta_{z}=16.25 \mathrm{~mm}, G_{2}$ has stepsizes $\Delta_{r}=12.5$ $\mathrm{mm}, \Delta_{z}=8.12 \mathrm{~mm}$. The shift-factors are $s_{1}=0.89, s_{2}=0.97$. Without shift the eigenvalues $\lambda_{2}$ and $\lambda_{3}$ have positive real parts on $G_{2}$ and negative real parts on $G_{1}$. This constellation would cause grave convergence problems in multigrid iteration. With shift they have positive real parts on both grids.

The second measure to improve convergence is the under-interpolation. Figure $i$ compares the relative defect over the number of $\mathrm{V}$-cycles on grid $G_{2}$ for $3500 \mathrm{MHz}$. The advantage of the under-interpolation with $w=0.9425$ is obvious.

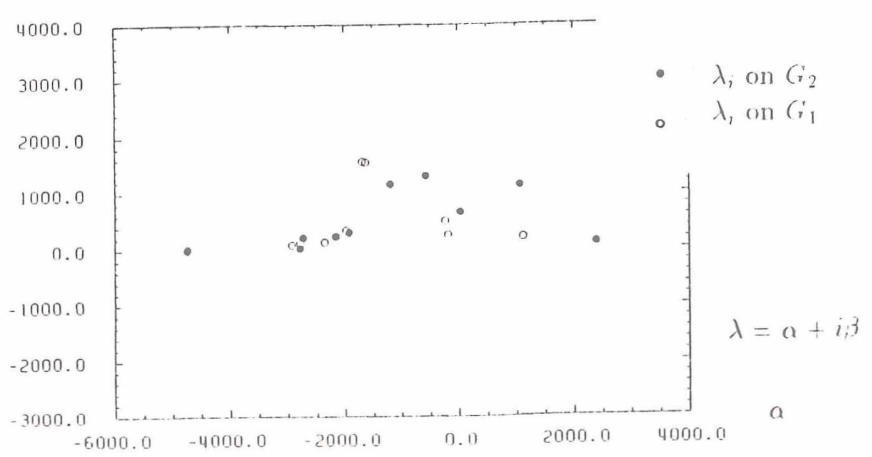

Figure 5: Lowest ten eigenvalues of $\mathbf{L}$ (without shift-factor) on the grids $G_{1}$ and $G_{2}$ for the pill-box and $3500 \mathrm{MHz}$.

ii

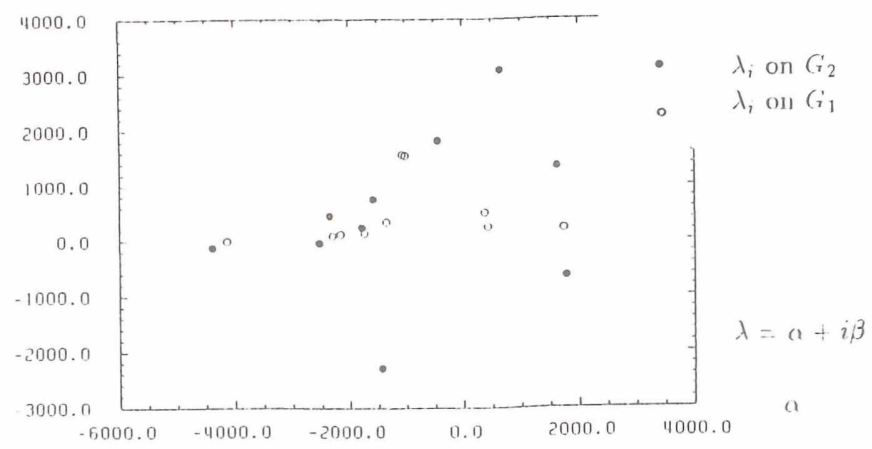

Figure 6: Lowest ten eigenvalues of $\mathrm{L}^{\mathrm{s}}$ (with shift-factor) on the grids $G_{1}$ and $G_{2}$ for the pill-box and $3500 \mathrm{MHz}$.

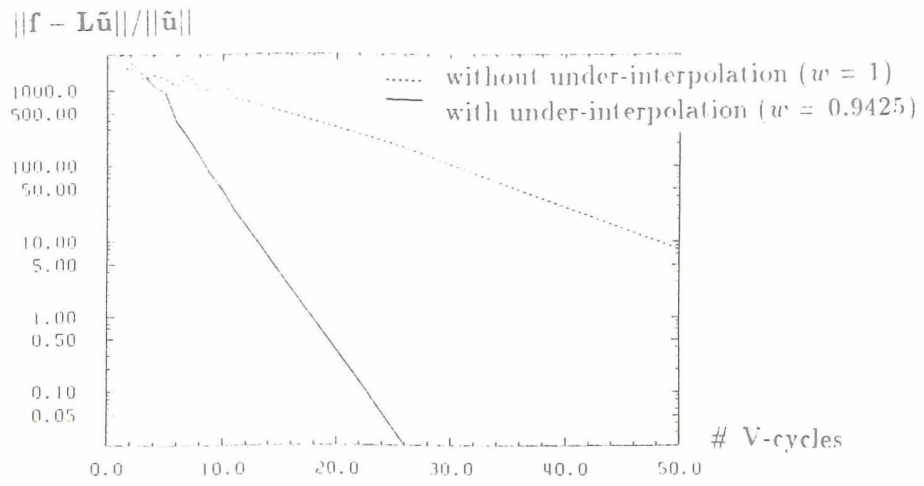

Figure $i$ : Relative defect $\|f-L \tilde{u}\|_{2} /\|\bar{u}\|_{2}$ on the fine grid $G_{2}$ of a 2-level-iteration in dependance on the number of V-cycles for 3500 MIIz.

Another typical characteristic of the indefiniteness of the problem is the dependance of the convergence on the chosen frequency: While swecping through the frequencies the problem becomes ill-conditioned several times due to singularity of the matrix.

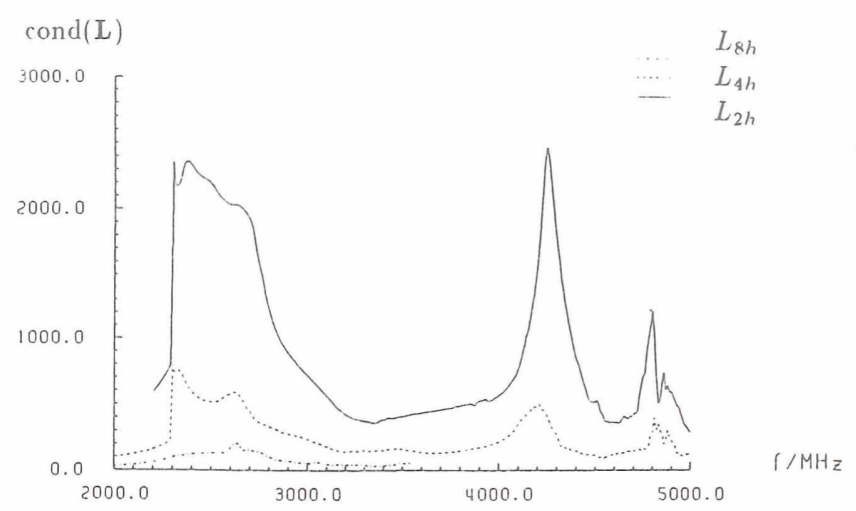

Figure 8: Condjtion number of $L_{2 h}, L_{4 h}, L_{8 h}$.

Figure 8 shows the condition number of $\mathrm{L}$ on three different grids. Three intervals with ill-condition can be observed. On grid $G_{8 h}$ computation only makes sense up to $3600 \mathrm{MHz}$.

In Figuse 9 the dependance of the relative defect on grid $G_{h}$ on the frequency is compared for the multigrid iteration on two, three and four levels. $G_{h}$ is always the finest grid level. As long as the stepsize is fine enough for the frequency range, the multigrid characteristic that the result only depends on the finest grid but not on the number of grid levels could be verified. This was also obtained by comparing results from LU-factorization and the multigrid algorithm. These comparisons were possible in the frequency range where a big $z$-stepsize is possible, i.e. $N$ is relatively small.

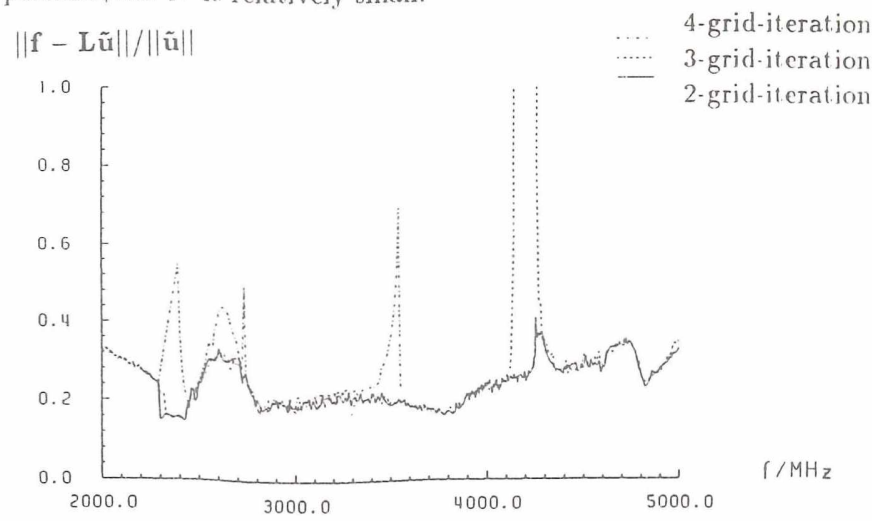

Figure 9: Relative defect. $\|f-L \bar{u}\|_{2} /\|\tilde{u}\|_{2}$ on $G_{h}$ in dependance on the frequency for a 2-, 3- and 4-grid-iteration. 
The following examples show the superconducting HERA cavity [13]. The fine grid has about 2000 points, the coarse grid of a 2-griditeration has about 500 points.

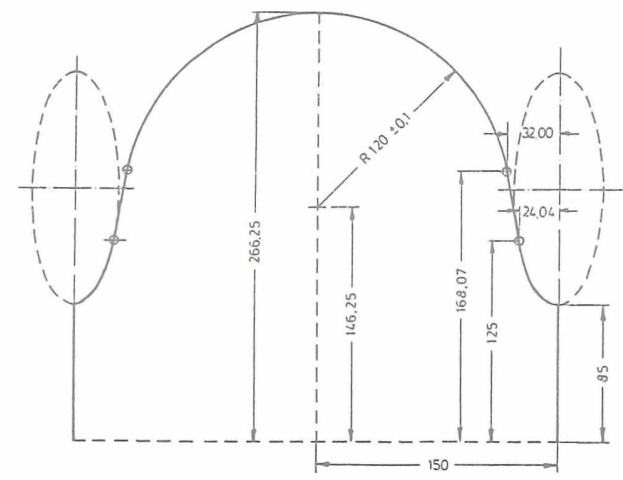

Figure 10: Superconducting HERA cavity

Below cut-off the results for the imaginary impedance have been verified with URMEL-T (see figure 11).

Figure 12 shows the real part of the impedance above cut-off. A typical attribute of the numerical problem is shown there: As the frequency increases a fincr and finer mesh is required, otherwise convergence problems may arise near a quasi-sesonance. C'omparision of the direct solution on the finer grid with results from TBCI + FFT show a very good agreement.

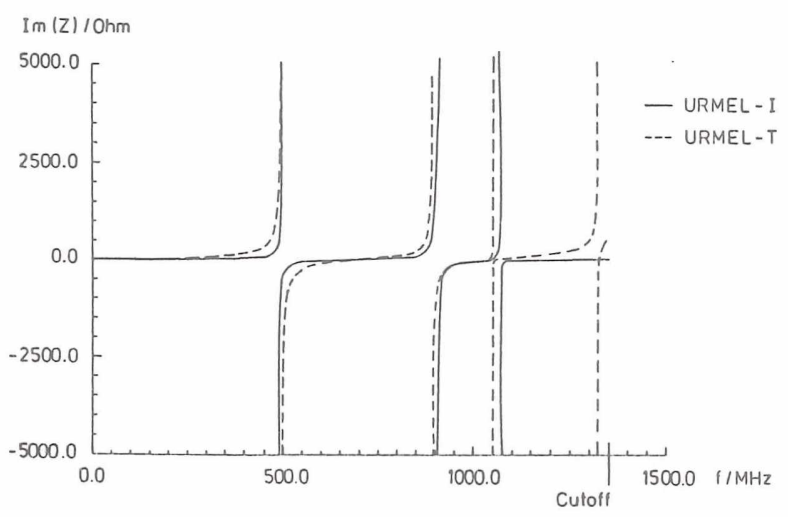

Figure 11: Comparision of imaginary part of the impedance below cut-off of the HERA-cavity by solution with URMEL-I and URMEL-T.

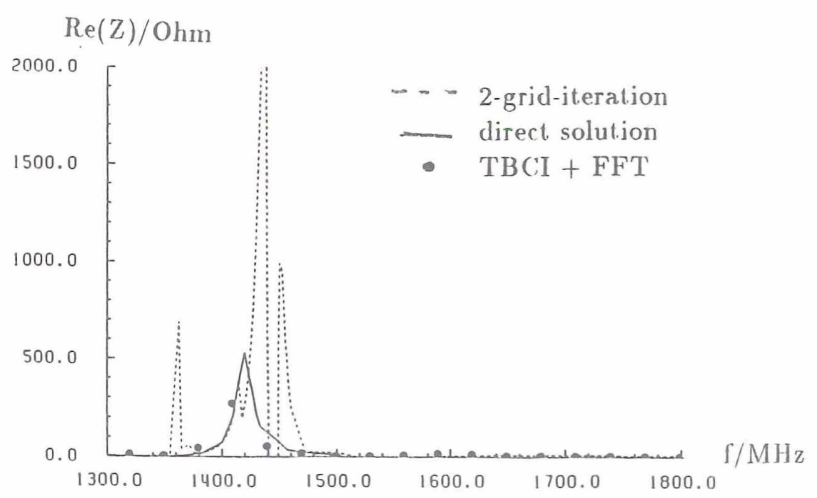

Figure 12: Comparision of real part of the impedance by direct solution on grid with 2145 points, 2-grid-solution on this grid and solution of TBCI + FFT for the HERA-cavity.
Finally the ruality factor of a quasj-resnmance ahove cut-off is compared for a cavily marde up from one. two and thee pill-bores with the dimensions given in the first example. The following approximate results were obtained:

\begin{tabular}{|l|r|r|c|}
\hline & $\mathrm{f} / \mathrm{MHz}$ & $\Delta \mathrm{f} / \mathrm{MHz}$ & \multicolumn{1}{c|}{ ? } \\
\hline one cell & 2640 & 200 & 13 \\
two cells & 2590 & 11 & 240 \\
three cells & 2580 & 5 & 506 \\
\hline
\end{tabular}

\section{S(MMAR)}

The code URMEL-I presents a now tool to calculate the impedance of obstacles of arbitrary but cylindrically symmetric shape with side tubes. The present version gives good result.s, indicating the validity of the method. URMEL-I is especially characterized by the use of the FIT-method which guarantees physical solutions and by the new multigrid algorithm for the fast oscillating, indefinite algebraic problem.

\section{ACKNOWLEDGEMENTS}

The authors wish to thank B. Steffen from the Kernforschungsanlage Jülich for many useful suggestions in connection with the multigrid algorithm and S.G.Wipf for careful reading of the manuscript.

\section{REFERENCES}

[1] T.Weiland, NIM vol. 216, 329-348 (1983)

[2] U.van Rienen,T.Weiland, Part. Acc. vol. 20, 239-266 (198i)

[3] K.Halbach,R.F.Holsinger, Part. Acc. vol. 7., 213-222 (1976)

[4] R.L.Gluckstern,F.Neri, in Intern. High Energy Acc. ('onf., Novosibirsk,vol.2, 170-173 (1986)

[5] U.van Riencn, T. Weiland, in Lin. Acc. Conf., 1986 SLAC:-Report-303, 289-291

[6] T.Weiland, NIMI vol. 216, 31-34 (1983)

[7] T.Weiland, $\underline{A E Z ̈} 31$ (197T), 116-120

[8] W.Hackbusch. Springer Series in Computational Math. 4, 1985

[9] S.Kaczmarz, Bull.Int.Acad.Polon.Sci. A 1937, 355-357 (193i; Zbl.1i, 31T)

[10] J.J.Dongarra et.al., SIAM, Philadelphia, 1979

[11] U.van Riemen. DESY-M-89-01, June 1989

[12] H.Henke, C:ERN-LEP-RF/85-41,Nov. 1985

[13] B.Dwersteg et al., DESY-M-88-04, August 1988 